\title{
The Role of Avatars in e-Government Interfaces
}

\author{
Badr Almutairi \\ Faculty of Technology, De Montfort University, Leicester LE1 9BH, UK \\ Badr@dmu.ac.uk \\ Dimitrios Rigas \\ University of West London, London W5 5RF, UK \\ Dimitrios.Rigas@uwl.ac.uk
}

\begin{abstract}
This paper investigates the use of avatars to communicate live message in e-government interfaces. A comparative study is presented that evaluates the contribution of multimodal metaphors (including avatars) to the usability of interfaces for e-government and user trust. The communication metaphors evaluated included text, earcons, recorded speech and avatars. The experimental platform used for the experiment involved two interface versions with a sample of 30 users. The results demonstrated that the use of multimodal metaphors in an e-government interface can significantly contribute to enhancing the usability and increase trust of users to the e-government interface. A set of design guidelines, for the use of multimodal metaphors in e-government interfaces, was also produced.
\end{abstract}

Keywords: multimodal, e-government, interfaces, avatar, speech, earcons, trust

\section{INTRODUCTION}

This research involves an empirical exploration to investigate usability aspects of e-government interfaces that incorporate a combination of multimodal metaphors. This study evaluates the contribution of these metaphors to usability, ease of communication and user trust. An e-government experimental platform, with two interface versions, was developed to serve as a basis for this investigation. The platform uses an input interface to send messages and an output interface to receive messages. Two groups of users were used to evaluate the results in terms of efficiency, effectiveness, user satisfaction and perception of trust.

\section{LITERATURE REVIEW}

\subsection{Multimodal Interaction}

"The auditory channel, as a whole, has been neglected in the development of userinterfaces, possibly because there is very little known about how humans understand

adfa, p. 1, 2011.

(C) Springer-Verlag Berlin Heidelberg 2011 
and process auditory stimuli" [1]. Interfaces that offer interaction using more than one sense are highly demanded. This is called multimodal metaphors. Rigas et al, suggest that the use of multimodal metaphors in application interfaces can be more useful to communicate the information that needs to be communicated to the user [2]. Also, they found that the use of speech and non-speech in interface application helped the users to make fewer mistakes and reduced the time taken when accomplishing their tasks [3]. Other several studies have been carried out to test the use of multimodal metaphors in visual user interface and to evaluate and examine the effect of these metaphors on the usability of computer applications [4 and 5].

\subsection{Usability evaluation e-government interfaces}

Usability is one of the most important factors to evaluate Human-Computer Interaction [6] and software quality [7]. It can be defined as the "extent to which a product can be used by specified users to achieve specified goals with effectiveness, efficiency and satisfaction" [8]. The effective system can be implemented and developed only by understanding the government websites, expectations of the users' under the citizen-centric approach, and the barriers that might hinder these Websites to provide the desired services through the Internet [9]. This technology can be used to improving the efficiency of governments; improving interaction between government and public, facilitating economic development, reducing costs, and meeting citizens' expectations for service delivery, by facilitating the process of administrative procedures [10 and $11]$.

\subsection{Trust factor}

Throughout the social sciences, trust is recognized as an important factor that mediates many aspects of human behavior [12, 13 and 14] Definitions of trust vary but a widely accepted one is that it is "a psychological state comprising the intention to accept vulnerability based upon positive expectations of the intentions or behavior of another" [15]. Thus, a person (the trustor) who depends on someone else (the trustee) expects to reduce the likelihood or size of a negative outcome in some situation: when that dependence is misplaced, the expected value of the outcome is lower. The online environment does not allow the natural benefits of face-to-face communications and to directly observe the service provider's behavior, which happens to be an assurance mechanism on which humans have depended on for ages. Based on trust, new service paradigms could emerge, developing passive citizen participation into active citizen participation in public service delivery [22]. As a number of features of online communication have the ability to both decrease or increase the level of citizens trust, it would be valuable to understand which factors and what levels will have desirables effect and which wouldn't. This will then help with ensuring that these factors are executed in such a manner that ensures that citizens can place the optimal level of trust in e-government. 


\section{$3 \quad$ AIMS}

The main aim of this research was to measure the impact of combining recorded natural speech and earcons and avatar on the usability of e-government interfaces. It also aimed to evaluate the extent to which the addition of these multimodal metaphors can affect the ability to communicate with users. More specifically, this experiment is aimed at testing the efficiency, effectiveness and user satisfaction of a multimodal egovernment interface, as opposed to a typical text based interface. In general, this experiment is aimed at investigating the usability aspects and communication performance of e-government interfaces that combine text and recorded speech and earcons to improve trust between users and government. In other words, this study is aimed at exploring if the addition of the aforementioned multimodal metaphors can result in a significant enhancement in terms of efficiency, effectiveness, satisfaction and trust of e-government interfaces.

\section{OBJECTIVES}

Through the aims stated, the following objectives were considered in the formulation of the hypothesis and development of the experimental platforms that present the deliver the message in e-government interfaces. An experimental investigation was undertaken into the role of expressive avatars in e-government interfaces using the experimental platforms developed by one group of users. The efficiency of the platforms tested was measured by recording the time users spent in completing the required tasks. The effectiveness of the platforms tested was also measured by calculating the percentage of message tasks correctly completed by users. This measure was also used to assess the communication performance of users. User satisfaction was tested by rating different aspects of the platforms tested. 


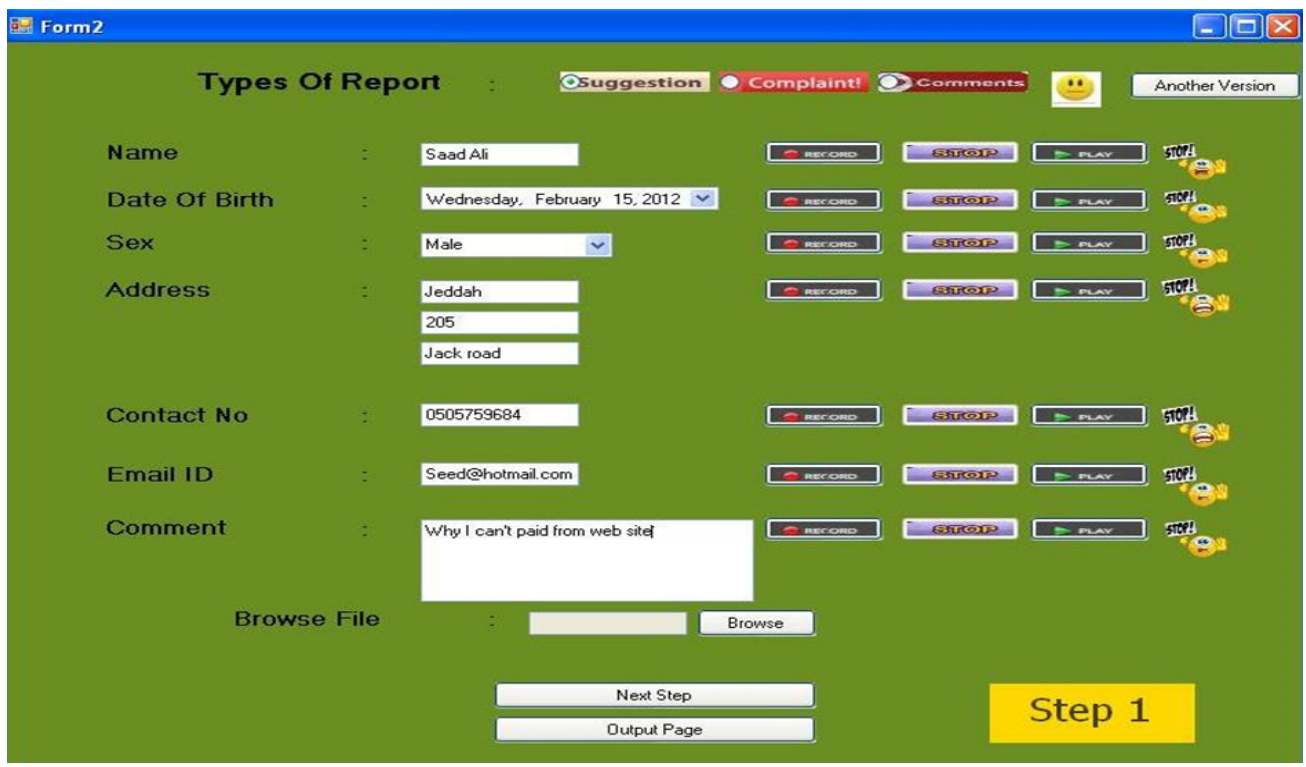

Fig. 1. Multi-Modal e-government Platform (MMEGP) input interface by incorporating auditory stimuli

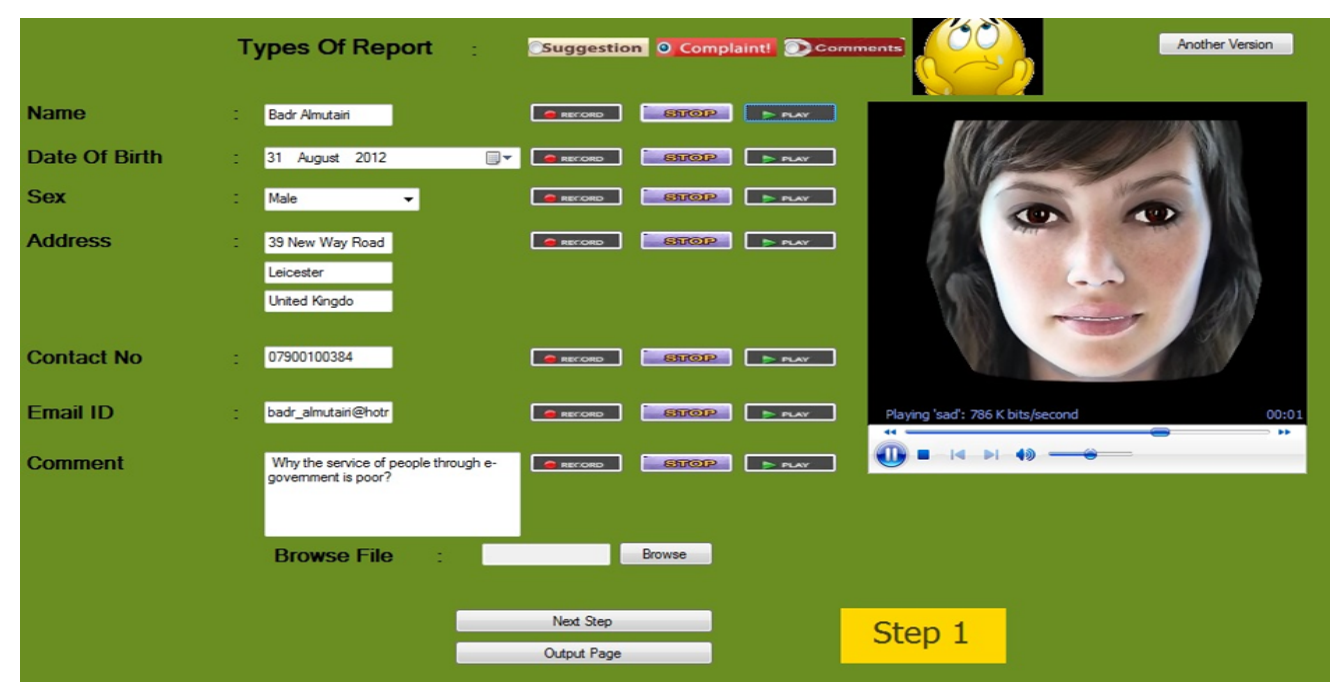

Fig. 2. Avatar e-government Platform (AVEGP) input interface by Avatar

\section{EXPERIMENTAL E-GOVERNMENT PLATFORM}

E-government platform was specially developed for this empirical investigation. The platform provided two different interface versions and were built from scratch 
and designed to utilize speaking avatars with human-like facial expressions, and a multimodal version. Both interface versions of the experimental platform were designed to deliver the same information about software representation of a given message statement and each interface was divided into an Input and Output. The software, in the form of three message types (Suggestion, Complaint and Comment), included explanations about Specific requests. There are three examples of common message types with different complexities (easy, moderate, complex). The complexity of these examples was gradually increased. In addition to question type, this study also investigated the effect of two types of evaluation questions; recall and recognition on the usability of the e-government interfaces tested, as well as on users' performance in terms of the output interface's property.

\section{VARIABLES}

The variables considered in the experimental design can be classified into three types which are: independent variables, dependent variables and controlled variables.

\begin{tabular}{llllll}
\hline Variable Code & Variable & Levels & State 1 & State 2 & State 3 \\
\hline IV 1 & Communication method & 2 & MMEGP & AVEGP & \\
\hline IV 2 & Message complexity & 3 & Easy & Moderate & Difficult \\
\hline IV 3 & Message type & 3 & Suggest & Complain & Comment \\
\hline
\end{tabular}

Table 1. Independent variables considered in the experiment (Input interface)

\begin{tabular}{llllll}
\hline Variable Code & Variable & Levels & State 1 & State 2 & State 3 \\
\hline IV 4 & Communication method & 2 & MMEGP & AVEGP & \\
\hline IV 5 & Message complexity & 3 & Easy & Moderate & Difficult \\
\hline IV 6 & Message type & 3 & Suggest & Complain & Comment \\
\hline IV 7 & Question type & 2 & Recognition & Recall & \\
\hline
\end{tabular}

Table 2. Independent variables considered in the second experiment (Output interface)

\begin{tabular}{lll}
\hline Variable Code & Variable & Measure \\
\hline DV 1 & Tasks messaging and question answering time & Efficiency \\
& & \\
\hline
\end{tabular}




\begin{tabular}{lll}
\hline DV 2 & Correctness of enter tasks and answers & $\begin{array}{l}\text { Effectiveness and user's } \\
\text { performance }\end{array}$ \\
\hline DV 3 & User satisfaction & Satisfaction \\
\hline DV 4 & User trust & perception of trust \\
\hline
\end{tabular}

Table 3. Dependent variables considered in the second experiment

\section{DATA COLLECTION}

The data collection process was based on experimental observations and questionnaires. For each task, each user was required to complete nine message tasks and to answer six questions. The time spent to complete the message tasks and to answer each of the six questions was recorded to help measure the efficiency. However, in order to collect the data related to effectiveness, the correctness of users' answers was checked and the total number of successful users who completed the message tasks and answered questions was counted for each user. The pre-experimental part of the questionnaire was dedicated to gathering personal data about users such as age, gender and education. It also helped to obtain data related to users' prior experience in computers, Internet and e-government. Finally, the post-experimental part of the questionnaire was aimed at assessing the users' satisfaction with the e-government platform tested. Users' responses to this questionnaire were used to calculate the satisfaction score for each user in both the control and the experimental groups.

\section{IMPLEMENTATION OF AVATARS}

An avatar is another multimodal interaction metaphor that could involve both visual and auditory human senses. It is a computer-based character that has been utilized to virtually represent one party in an interactive context $[16,17]$ with the ability to communicate verbal and non-verbal information [18, 19]. Verbal communication refers to the use of speech and written messages whereas nonverbal communication can be attained by facial expressions [20]. In general, avatars can be classified as abstract, realistic and naturalistic.

An avatar is a software or tool which can be used to allow users to speak about something through live message formats which can be used to give complaints, suggestions or comments. Use of the avatar is compared with video messages or recorded messages during this study. The efficiency and effectiveness of the messages can be determined by the time factor and by the number of mouse clicks as well as user satisfaction. 


\section{EFFICIENCY}

The time taken to perform tasks and answer the required questions was used as a measure of efficiency. This measure was considered for all tasks for the input interface and for the output interface (according to the question type, recall and recognition), message complexity, as well as for each task and for each of the users in both groups.

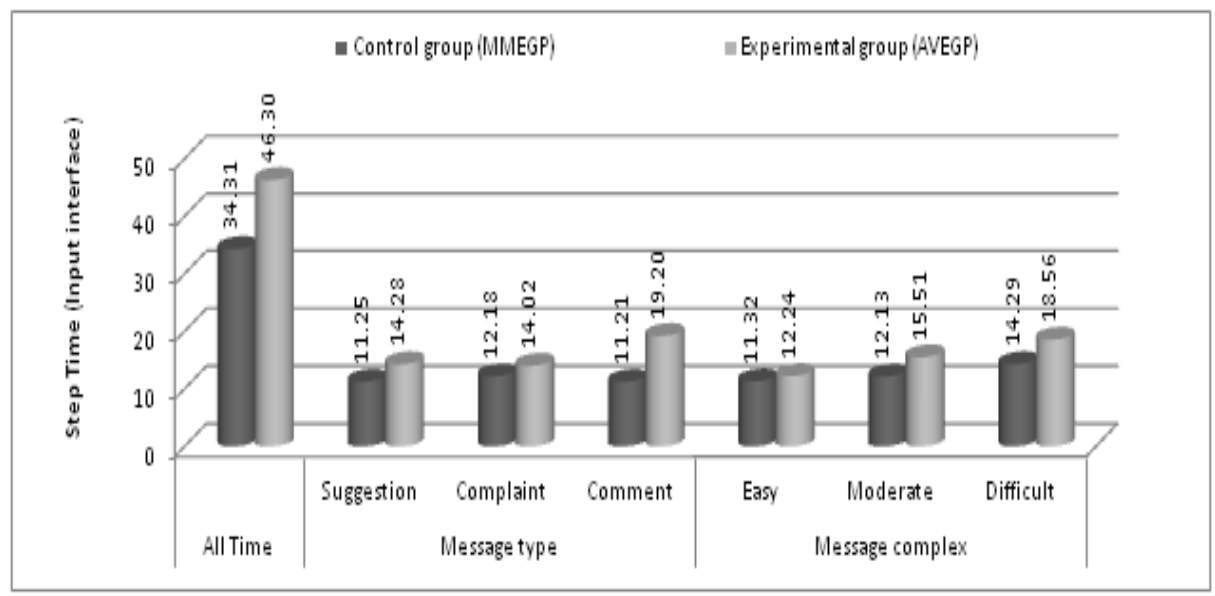

Fig. 3. Mean values of time taken by users in both groups to enter all tasks, (Input interface)

The control group spent a total of 34.3 minutes but note that the experimental group spends more time, 46.3 minutes, because they must enter both text and avatar video for each task. Figure 3 shows the mean values of the time taken by all users.

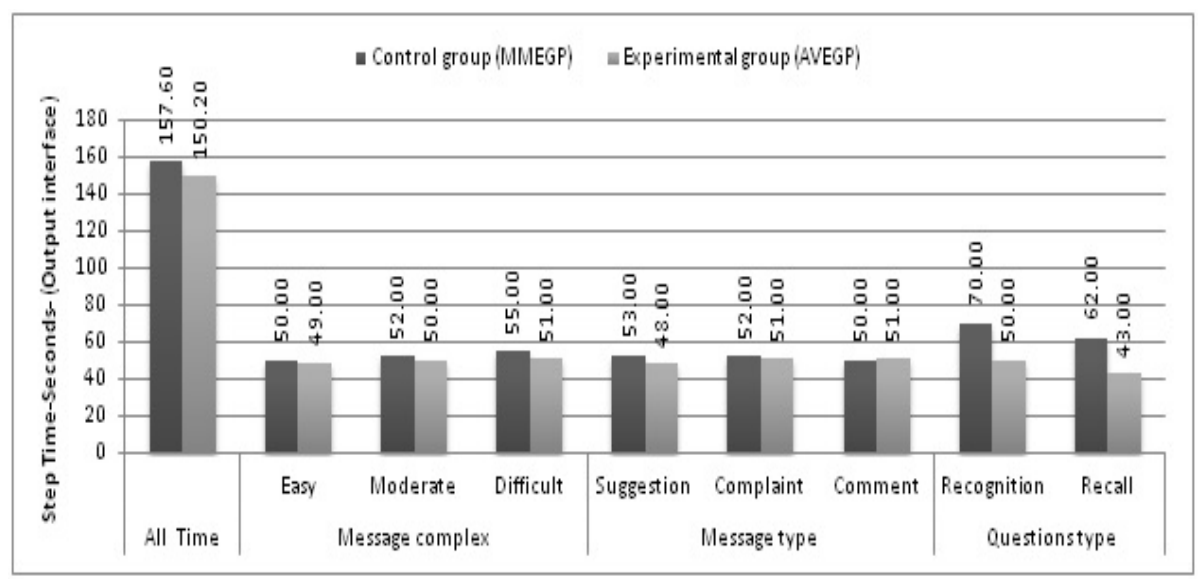

Fig. 4. Mean values of time taken by users in both groups to enter all tasks, (Output interface) 
The experimental group Figure 4 took less time to complete the tasks. It can be said the use of avatar video improved efficiency, as tasks took less time - unlike the other groups which took more time to listen and read the tasks in the output interface.

\section{Effectiveness}

The number of correctly entered messages were used as a measure of effectiveness. This measure was considered for all messages and all the questions, according to the question type (recall and recognition) and message complexity (easy, moderate and difficult) and message type (suggestion, complain and comment), as well as for each user in both control and experimental groups.

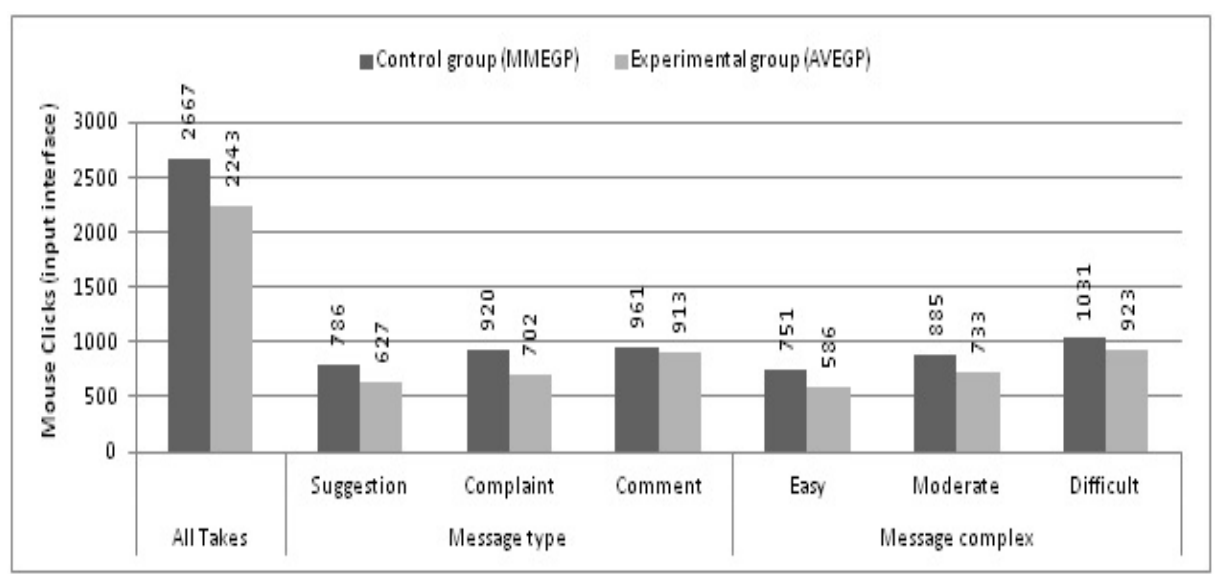

Fig. 5. The mean number of mouse clicks performed by users in both groups to enter messages for all the tasks for the input interface.

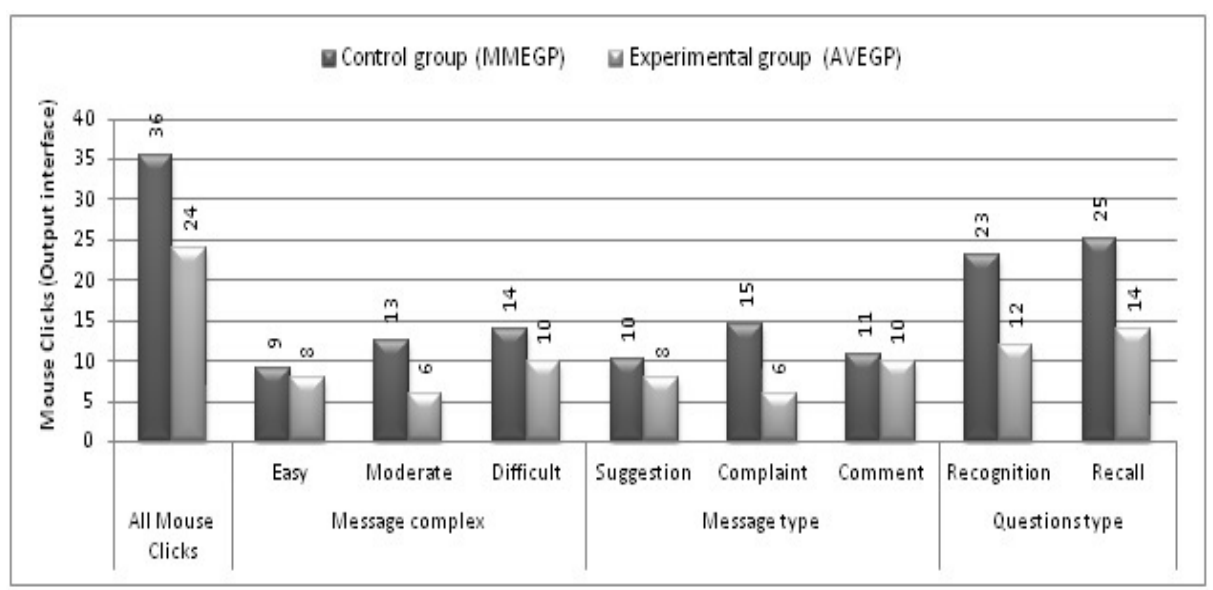


Fig. 6. The mean number of mouse clicks by users in both groups to enter message for all the tasks in the output interface.

Figure 5 shows the percentage of mouse clicks to enter messages for all tasks for the MMEGP and AVEGP. It can be noted that users of the AVEGP used less mouse clicks that users of the MMEGP. The reason for this is the enhanced input interface used by users when using the new avatar tool. The mean number of mouse clicks for the MMEGP was (2667) more than that for the AVEGP (2243), for all messages. The t-test results revealed that the difference in mouse clicks between MMEGP and TOEGP was significant ( $\mathrm{t}(23), \mathrm{MD}=624, \mathrm{p}<0.05)$.

As a result, the AVEGP users outperformed the users of the MMEGP Figures 6, 7 explain that, who send the messaging information via the text channel and avatar.

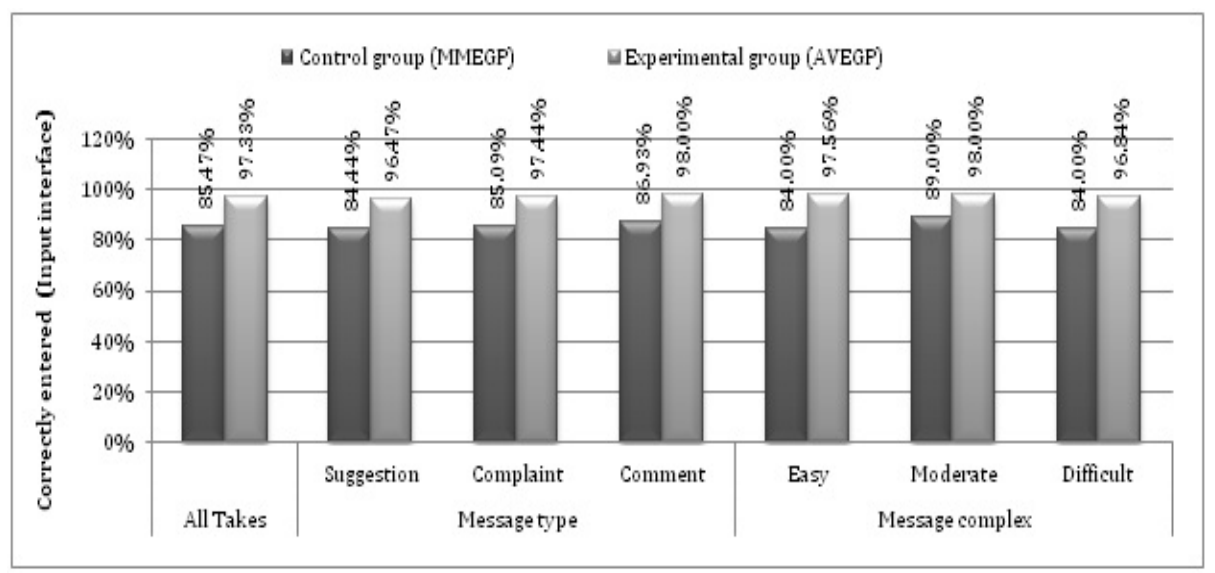

Fig. 7. Percentage of correctly completed tasks for users in both groups for the (output interface).

\section{User Satisfaction}

Users' responses to SUS questionnaire (10 statements) was used to measure their attitude after they had the opportunity to use each experimental platform. The overall satisfaction score for each user was calculated using the SUS (System Usability Scale) method [21]. Through Figure 8 the mean satisfaction score for the users in the AVEGP was more satisfactory for users using the MMEGP. 


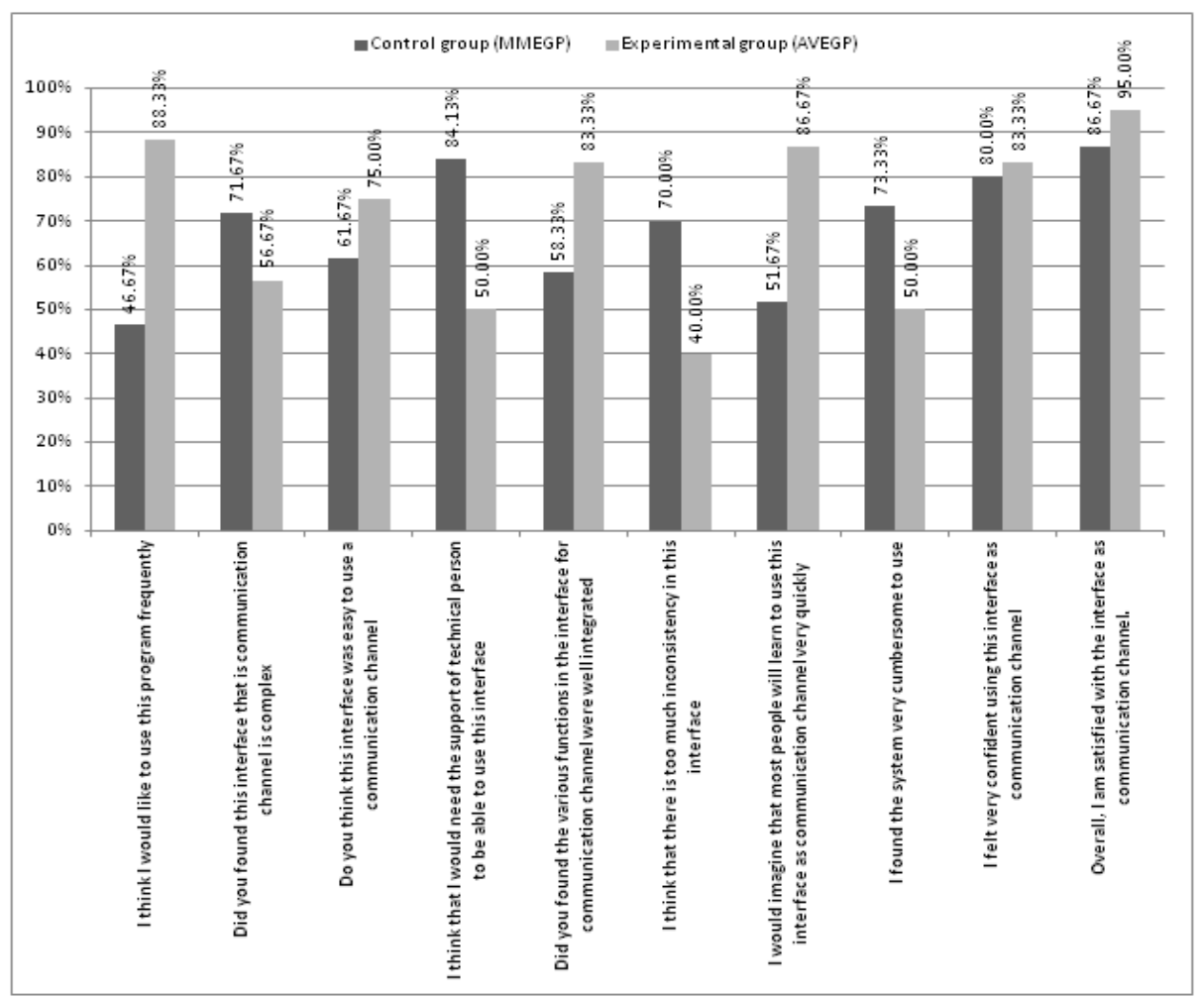

Fig. 8. Percentage of users agreeing to each statement of satisfaction, for both MMEGP and AVEGP groups.

\section{Perception of Trust}

Figure 9 shows the chart of user agreement and disagreement with the trust statements for using the MMEGP and AVEGP experimental structures.

In general, participants responded in favour of AVEGP with respect to the five aspects of trust this shows when using multimodal approach and then increase trustworthy. 


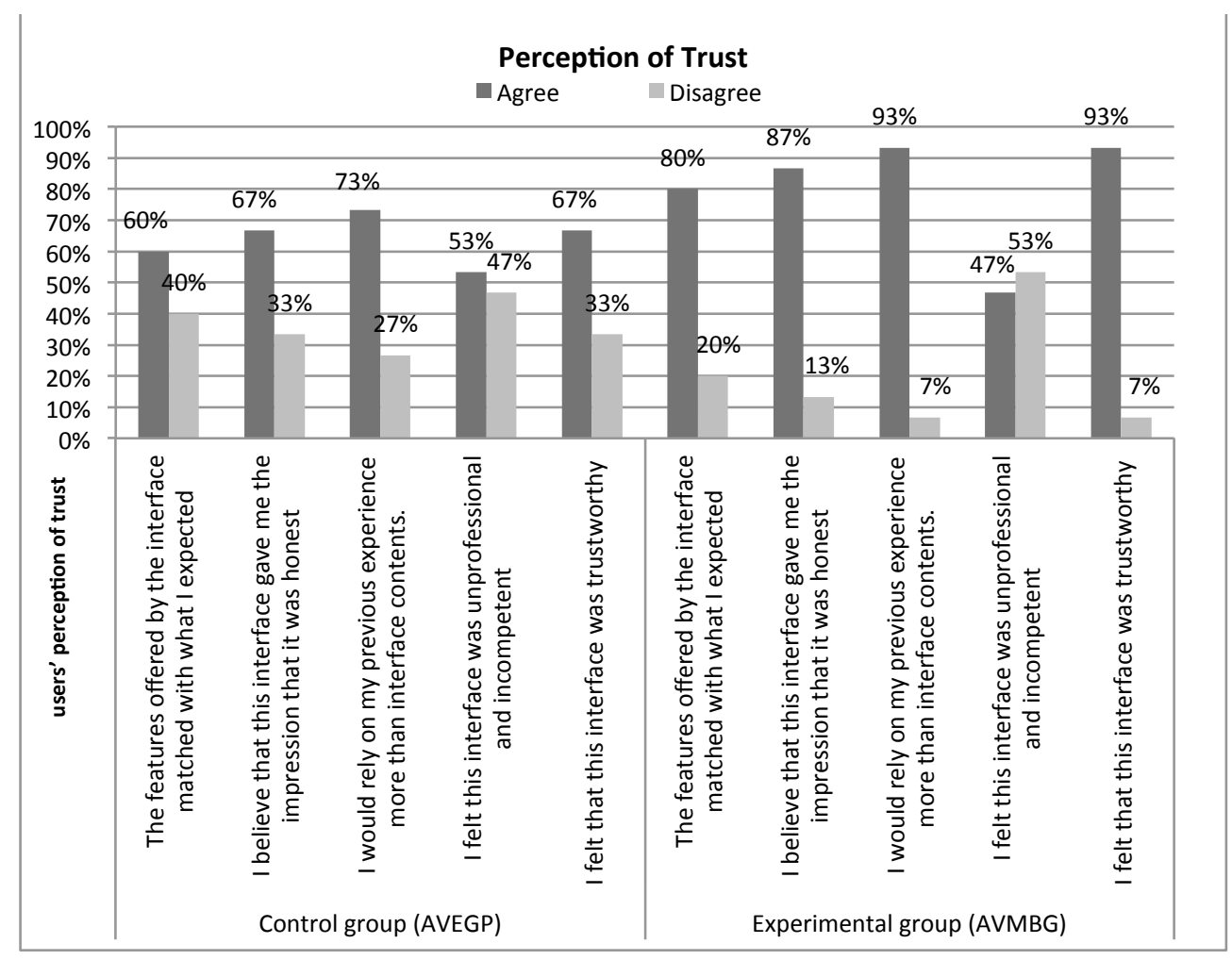

Fig. 9. Percentage of users agreeing and disagreement to each trust statement, for both MMEGP and AVEGP groups.

\section{DISCUSSION}

The current study investigated the usability and communication performance of a new multimodal e-government interface with avatar, as opposed to a recorded speech based one. The results obtained have been used to compare the two interfaces in terms of efficiency, effectiveness, user satisfaction and user trust. The study also focused on the factors that can affect the role of multimodal interaction metaphors, such as the message type (suggestion, complaint, comment) the message complexity level (easy, moderate and difficult) and the question type (recall and recognition).

On the whole, it was expected that users of the AVEGP would be more satisfied than the users of the MMEGP. Consistent with this assumption, the multimodal presentation of the message content in the AVEGP was shown to be significantly more satisfactory than the record speech interface in the MMEGP. It seems that using the avatar approach is interesting and attractive for users in the experimental group, as they expressed a more positive attitude towards the video-visual communication of the mes- 
sage content. Although both of the e-government interfaces tested were easy to use, neither was confusing or nervous. The results obtained did not demonstrate a remarkable difference between both groups of users regarding these satisfaction features (refer to Q1 to Q6 in Figure satisfaction. However, a larger difference was observed for statements related to communication (refer to Q7 to Q10 in Figure 19). These results derived from two independent groups and users within those two groups and were not presented for both interface versions in order to make an informed comparison. However, users in the experimental group might have had prior experience to typical government interfaces and this possibly served as a comparison point. Naturally, users in the experimental group thought that their communication with the government was improved and aided by the multimodal metaphors. It was easier for them to identify the messaging information, which was communicated by the avatar. This result on its own is not conclusive; as it is based on subjective rating of users and the typical mean difference is not large enough (even when a statistical significance for the overall satisfaction results was reached). Experiment show when using multimodal approach and increase Perception of Trust. In addition achieved the aims and the hypothesis in this experiment it found the participants in the experimental group expressed positive attitudes towards the nonverbal communication by avatar. The results suggested that users tend to be more comfortable with nonverbal communication when avatar conveyed more rapidly than for the first time. As the satisfaction, efficiency, effectiveness and perception of trust results are similar to each other, the argument that users in the experimental group were helped by the new multimodal metaphors becomes much stronger. It can be extrapolated that the multimodal aided egovernment is more likely to result in an agreeable and satisfying experience for the user. This experience is linked with the ability to complete message tasks correctly and quickly. In summary, the general results of this experimental study suggest the importance of the new multimodal interaction metaphors tested in enhancing users' messaging performance and the usability of e-government interfaces in terms of efficiency, effectiveness, user satisfaction and user trust.

\section{CONCLUSION}

This paper examines the impact of new multimodal interaction metaphors for ease of use, in terms of efficiency, effectiveness and user satisfaction and the communication performance of the e-government interfaces. This study has been implemented by developing two different versions of the experimental e-government platform. The first version was based on the use of recorded speech and earcons with text to present the messaging content between the sender and receiver. The second version was concerned with using a combination of new multimodal metaphors (avatar and text) to supply the same messaging content. Together, e-government platforms were then empirically evaluated by two independent groups of users. The first group (control) tested the recorded speech and earcons with a text interface by performing common tasks and answering a set of message evaluation questions. The second one (experi- 
mental) tested the new multimodal interface by performing common tasks and answering a set of message evaluation questions.

The results obtained from this experiment confirm that multimodal metaphors do in fact help to improve the usability of e-government interfaces, and reduce the time needed for users to respond to messages and, allow users to undertake activities more accurately, and make use of the interface more pleasing and satisfactory and more trustworthy. In other words, we conclude that the new multimodal metaphors tested can contribute greatly to improving the performance of users' communication and ease of use of e-government interfaces in terms of effectiveness and a percentage of $14 \%$ compared to the other group in input interface and $30 \%$ in the output interface, efficiency a percentage of $24 \%$ compared to the other group in input interface and $4 \%$ in the output interface and user satisfaction a percentage of $9 \%$ compared to the other group. It is therefore proposed to include multimodal metaphors in e-government interfaces and these needs to be taken in mind when designing such interfaces.The egovernment interface is gaining the popularity among the providers of e-government services. Its importance from the users' point of view has become the main concern for e-government service providers. This is why this paper has focused on investigating the usability which is an important factor for the improvement of e-government interfaces for the provision of high quality government services.

\section{REFERENCES}

1. D. I. Rigas, Guidelines for Auditory Interface Design: An Empirical Investigation. 1996: PhD thesis, Loughborough University of Technology.

2. D. Rigas, and D. Hopwood, The Role of Multimedia in Interfaces for OnLine Learning. 9th Panhellenic Conference on Informatics (PCl'2003). , Thessaloniki, Greece, 2003.

3. S. Oviatt, "Multimodal Interfaces," The Human-Computer Interaction Handbook: Fundamentals, Evolving Technologies, and Emerging Applications, 2003.

4. K. Al-Omar, and D. Rigas, An Empirical Investigation of different Interactive Environments in E-Commerce. In Proceedings of the Ninth Informat- 
ics Workshop for Research Students, Bradford, UK, p.p. 68-71, June 2008, ISBN: 978-1-85143-251-6.

5. M. M. Alsuraihi, and D. Rigas, Multimodal Interaction Metaphors for Interface Design: an Empirical Investigation on Learnability. Proceedings of the Saudi Innovation Conference 2007, Newcastle University, p.p. 686694, May 2007, ISBN: 978-0-955104-92-3.

6. J. Nielsen, Usability Engineering: Academic Press Inc., US, 1993.

7. M. F. Costabile, "Usability in the Software Life Cycle," Handbook of software engineering and knowledge engineering, vol. 1, pp. 179-192, 2001.

8. ISO, "ISO 9241: Ergonomic Requirements for Office Work with Visual Display Terminals (VDTs) - Part 11: Guidelines on usability," pp. 2, 1998.

9. A. Ciuffreda, "An Empirical Investigation in Using Multi-modal Metaphors to Browse Internet Search Results," in Department of Computing, School of Informatics, vol. PhD. Bradford, UK: University of Bradford, 2008.

10. M. Alotaibi, "Electronic Customer Knowledge Management Systems: a Multimodal Interaction Approach," in Informatics Research Institute, vol. PhD. Bradford, UK: University of Bradford, 2009.

11. D. I. Rigas, and D. Memery, and D. Hopwood, and M. A. Rodrigues, "Empirically derived design issues in auditory information processing for mobile telephony," Information Technology: Coding and Computing, 2000. Proceedings. International Conference on, pp. 462-469, 2000.

12. C. F. Camerer, (2003). Behavioral Game Theory. NewYork, N.Y: Russell Sage Foundation. 
13. F. Fukuyama, (1996). Trust: The Social Virtues and the Creation of Prosperity. London, UK: Penguin Books.

14. Kramer, R. M. and Tyler, T. R. (1996). Trust in Organizations: Frontiers of Theory and Research. London, UK: Sage

15. I. Markova, (Ed.) (2004). Trust and Democratic Transition in Postcommunist Europe. Oxford, UK: Oxford University Press.

16. D. M. Rousseau, S. B. Sitkin, R. S. Burt, and C.Camerer, (1998). Not so different after all: A cross-discipline view of trust. Academy of Management Review, 23, 393-404.

17. C. Bartneck, and T. Takahashi, and Y. Katagiri, "Cross Cultural Study of Expressive Avatars," Proceedings of the Social Intelligence Design, 2004.

18. M. D. Dickey, "3D virtual worlds: an emerging technology for traditional and distance learning," Proceedings Ohio Learning Network: The convergence of learning and technology - window on the future oln.org/conferences/OLN2003/papers/Dickey3DVirtualWorlds. pdf, 2003.

19. J. Beskow, "Animation of Talking Agents," Proceedings of AVSP, vol. 97, pp. 149-152, 1997.

20. R. Sheth, "Avatar Technology: Giving a Face to the e-Learning Interface," The eLearning Developers' Journal, 2003.

21. J. Brooke, "SUS: a" quick and dirty" usability scale," Usability evaluation in industry, pp. 189-194, 1996. 
22. S. E. Colesca, "Understanding trust in e-government," Inzinerine Ekonomika-Engineering Economics, vol. 3, pp. 7-15, 2009. 